\title{
Thermal Modifications of the Heavy Axial Vector Mesons Properties
}

\author{
E. Veli Veliev* \\ Department of Physics, Kocaeli University, 41380 Izmit, Turkey \\ E-mail: Elsenakocaeli.edu.tr
}

K. Azizi

Department of Physics, Doğuş University, Acıbadem-Kadıköy, 34722 Istanbul, Turkey

E-mail: kaziziedoqus.edu.tr

\section{H. Sundu}

Department of Physics, Kocaeli University, 41380 Izmit, Turkey

E-mail: havrive.sundudkocaeli.edu.tr

\section{G. Kaya}

Department of Physics, Kocaeli University, 41380 Izmit, Turkey

E-mail: qulsahbozkirdkocaeli.edu.tr

\begin{abstract}
We investigate the properties of the heavy axial vector $\chi_{b 1}$ and $\chi_{c 1}$ quarkonia at finite temperature. Taking into account the thermal spectral density as well as additional operators coming up at finite temperature and perturbative two-loop order corrections to the correlation function, we obtained the thermal QCD sum rules for considering particles. It is observed that the masses and decay constants almost remain unchanged with respect to the variation of the temperature up to $T \approx 100 \mathrm{MeV}$, however after this point, the decay constants decrease sharply and approach approximately to zero at critical temperature. This situation may be interpreted as a signal for deconfinement phase transition and our results at zero temperature are in good consistency with the existing experimental values.
\end{abstract}

Xth Quark Confinement and the Hadron Spectrum,

October 8-12, 2012

TUM Campus Garching, Munich, Germany

\footnotetext{
* Speaker.
} 


\section{Thermal Sum Rule for Heavy Axial Vector Quarkonia}

Investigation of the properties of heavy quarkonia such as bottomonium $(\bar{b} b)$ and charmonium $(\bar{c} c)$ in medium can give valuable information about the QCD vacuum. Since hadrons are formed in a region of energy that is very far from the perturbative region, some nonperturbative approaches are needed to calculate their parameters. Among the nonperturbative methods, the QCD sum rules approach [W] is one of the most attractive, applicable and powerful method. The thermal version of this approach was proposed by Bochkarev and Shaposhnikov [ []].

We start considering two-point thermal correlation function of axial vector currents:

$$
\Pi_{\mu v}(q, T)=i \int d^{4} x e^{i q \cdot x}\left\langle\mathscr{T}\left(J_{\mu}(x) J_{v}^{\dagger}(0)\right)\right\rangle,
$$

where, $J_{\mu}(x)=: \bar{Q}(x) \gamma_{\mu} \gamma_{5} Q(x)$ : with $Q=b$ or $c$ is the interpolating current of heavy axial vector meson, $T$ is temperature and $\mathscr{T}$ denotes the time ordering product. The aforesaid correlation function can be calculated both in terms of the hadronic parameters called the physical or phenomenological side, and in terms of the QCD parameters called the theoretical or QCD side. These two representations are matched using dispersion relations to obtain the QCD sum rules for the physical observables under consideration. After applying the Borel transformation to suppress the higher states and continuum contributions further, we get (for more details see also [3])

$$
f_{A}^{2}(T) m_{A}^{2}(T) \exp \left(-\frac{s}{M^{2}}\right)=\int_{4 m^{2}}^{s_{0}(T)} d s\left[\rho_{t, a}(s)+\rho_{\alpha_{s}}(s)\right] \exp \left(-\frac{s}{M^{2}}\right)+\widehat{B} \Pi_{t}^{n p},
$$

where the annihilation part of the spectral density is expressed as:

$$
\rho_{t, a}(s)=\frac{s}{4 \pi^{2}} v^{3}\left[1-2 n\left(\frac{\sqrt{s}}{2 T}\right)\right]
$$

and the perturbative two-loop $\alpha_{s}$ order correction to the spectral density at zero temperature is given by [U, 田]:

$$
\begin{aligned}
\rho_{\alpha_{s}}(s) & =\alpha_{s} \frac{s}{3 \pi^{3}}\left[\pi v^{3}\left(\frac{\pi}{2 v}-\frac{1+v}{2}\left(\frac{\pi}{2}-\frac{3}{\pi}\right)\right)-\frac{21}{16} v+\frac{30}{16} v^{3}+\frac{3}{16} v^{5}-\frac{3}{2} v\left(1+v^{2}\right)\right. \\
& \left.+\left(\frac{21}{32}+\frac{59}{32} v^{2}+\frac{19}{32} v^{4}-\frac{3}{32} v^{6}-\frac{5}{4}\left(1+v^{2}\right)^{2}+2\right) \ln \frac{1+v}{1-v}\right]
\end{aligned}
$$

where $v=v(s)=\sqrt{1-4 m^{2} / s}$ and $n(x)=[\exp (\beta x)+1]^{-1}$ is the Fermi distribution function. In order to obtain the thermal version of the two-loop $\alpha_{s}$ order correction, the strong coupling $\alpha_{s}$ is replaced with its temperature dependent lattice improved version given in [5]. Also, the nonperturbative part in Borel scheme is obtained as:

$$
\begin{aligned}
\hat{B} \Pi_{t}^{n p} & =\int_{0}^{1} d x \frac{\exp \left[\frac{m^{2}}{M^{2}(-1+x) x}\right]}{288 M^{6} \pi^{2}(-1+x)^{4} x^{4}}\left[3 \langle \alpha _ { s } G ^ { 2 } \rangle \left(8 M^{6} x^{4}(x-1)^{4}+m^{6}(1-2 x)^{2}\left(1-2 x+x^{2}\right)\right.\right. \\
& -m^{2} M^{4}(x-1)^{2}\left(2 x^{6}-4 x^{5}+4 x^{4}-2 x^{3}-3 x^{2}\right)+m^{4} M^{2}\left(8 x^{6}-24 x^{5}+13 x^{4}+14 x^{3}-14 x^{2}\right. \\
& +3 x))+4 \alpha_{s}\left\langle\Theta^{g}\right\rangle\left(m^{2} M^{4}(x-1)^{2}\left(22 x^{6}-44 x^{5}+74 x^{4}-52 x^{3}+x^{2}\right)-M^{6}(x-1)^{3}\left(4 x^{7}-8 x^{6}\right.\right. \\
& \left.++38 x^{5}-34 x^{4}+12 x^{3}\right)-3 m^{6}(1-2 x)^{2}\left(1-2 x+2 x^{2}\right)-m^{4} M^{2}\left(40 x^{6}-120 x^{5}+99 x^{4}+2 x^{3}\right. \\
& \left.\left.\left.-28 x^{2}+7 x\right)\right)\right]
\end{aligned}
$$

where $M^{2}$ and $m$ are the Borel mass parameter and quark mass, respectively. 


\section{Conclusion and Discussion}

The sum rules for the masses and decay constants contain two auxiliary parameters, namely continuum threshold $s_{0}$ and Borel mass parameter $M^{2}$. The continuum threshold $s_{0}$ is not completely arbitrary and it is related to the energy of the first exited state of the meson. The intervals of continuum thresholds for $\chi_{b 1}$ and $\chi_{c 1}$ heavy axial mesons are chosen as $s_{0}=(106-110) \mathrm{GeV}^{2}$ and $s_{0}=(15-17) \mathrm{GeV}^{2}$, respectively.

Taking into account the temperature dependencies of hadronic threshold, energy density, two loop $\alpha_{s}$ order correction, quark and gluon condensates, we obtained that decay constants of $\chi_{b 1}$ and $\chi_{c 1}$ obey following fit functions in unit of $\mathrm{GeV}$ :

$$
f(T)=A+B \exp (\lambda T)
$$

The values of the parameters $A, B$ and $\lambda$ are given in Table $\mathbb{D}$

\begin{tabular}{|c|c|c|c|}
\hline \hline & $A(\mathrm{GeV})$ & $B(\mathrm{GeV})$ & $\lambda\left(\mathrm{GeV}^{-1}\right)$ \\
\hline$\chi_{b 1}$ & 0.24 & $-2.33 \times 10^{-4}$ & 47.26 \\
\hline$\chi_{c 1}$ & 0.34 & $-7.94 \times 10^{-5}$ & 39.57 \\
\hline \hline
\end{tabular}

Table 1: Parameters appearing in fit function.

At $T=0$, values of the masses and decay constants for $\chi_{c 1}$ and $\chi_{b 1}$ are obtained as $m_{\chi_{c 1}}=$ $(3.52 \pm 0.11) \mathrm{GeV}, f_{\chi_{c 1}}=(0.344 \pm 0.027) \mathrm{GeV}, m_{\chi_{b 1}}=(9.96 \pm 0.26) \mathrm{GeV}, f_{\chi_{b 1}}=(0.240 \pm 0.012) \mathrm{GeV}$. The obtained mass results are in good consistency with the existing experimental data [目]. Our investigation shows that the masses and decay constants remain unchanged with the variation of temperature up to $T \cong 100 \mathrm{MeV}$, but after this point, they start to diminish with increasing temperature. At deconfinement temperature, the decay constants reach approximately to $22 \%$ of their vacuum values, while the masses decrease about $4 \%$, and $19 \%$ for $\chi_{b 1}$ and $\chi_{c 1}$ states, respectively.

\section{Acknowledgement}

This work has been supported in part by the Scientific and Technological Research Council of Turkey (TUBITAK) under the research project no. 110T284.

\section{References}

[1] M. A. Shifman, A. I. Vainstein, V. I. Zakharov, Nucl. Phys. B 147, 385 (1979); Nucl. Phys. $B$ 147, 448 (1979).

[2] A. I. Bochkarev, M. E. Shaposhnikov, Nucl. Phys. B268, 220, (1986).

[3] E. Veli Veliev, K. Azizi, H. Sundu, G. Kaya, arXiv:hep-ph/1205.5703

[4] L. J. Reinders, H. Rubinstein and S. Yazaki, Phys. Rep. 127, 1 (1985).

[5] E. Veli Veliev, K. Azizi, H. Sundu, G. Kaya, A. Turkan, Eur. Phys. J. A 47110 (2011).

[6] K. Nakamura et al., (Particle Data Group), J. Phys. G 37, 075021 (2010). 